\title{
Non-uniform deformation after prestrain
}

\author{
José-Valdemar Fernandes ${ }^{a}$, Luis F. Menezes ${ }^{\text {a }}$, Dulce M. Rodrigues ${ }^{\text {a }}$, Bruno M. Chaparro ${ }^{\text {a }}$, \\ Manuel F. Vieira ${ }^{b}$ \\ a Departamento de Engenharia Mecânica - CEMUC - Faculdade de Ciências e Tecnologia da Universidade de Coimbra, Pólo II da \\ Universidade de Coimbra - Pinhal de Marrocos, P-3030 Coimbra, Portugal \\ ${ }^{\mathrm{b}}$ Departamento de Engenharia Metalúrgica e Materiais - GMM/IMAT - Faculdade de Engenharia da Universidade do Porto, \\ Rua dos Bragas, P-4099 Porto Codex, Portugal
}

(Received 16 March 1999; revised 23 July 1999)

\begin{abstract}
The deformation behaviour of prestrained metal sheets is analysed in this work. The non-uniform deformation observed during reloading in tension was studied, by following deformation in different regions of the samples. It takes into account the presence of geometrical defects in the samples and explains the importance of mechanical behaviour. A simplified analysis was used, to model the behaviour in tension of a metallic specimen with geometrical imperfection. The flow behaviour is described using a Swift law equation, which includes strain-rate sensitivity. A modified law was used for prestrained materials and this incorporates the plastic prestrain value, adjusted to the path change. The model predicts imperfection growth kinetics with strain, and strain saturation in the homogeneous region, due to the onset of necking. (C) 2000 Éditions scientifiques et médicales Elsevier SAS

tensile deformation / plastic instability / complex strain paths
\end{abstract}

\section{Introduction}

During complex strain paths, the tensile instability and necking behaviour of metals presents some particular facets. From experimental results, a phenomenological model has been put forward (Vieira et al., 1990), in order to explain the occurrence, in some cases, of early plastic instability in tension after prestrain. It has been suggested (Schmitt et al., 1991; Fernandes et al., 1993) that the plastic instability, occurring in tension after prestrain, is a mechanical consequence of the drop in strain-hardening behaviour, which takes place at the beginning of reloading. Furthermore, macroscopic non-uniform deformation was clearly observed, at the beginning of reloading in tension of copper samples, prestrained up to low values $(\geqslant 0.06)$. No connection between this non-homogeneous deformation and the slip behaviour inside the grains was found. But, this can be explained by the flow behaviour in tension, which depends on the deformation history. In fact, when the strain path is changed during the plastic deformation of metals, the flow behaviour diverges from that which is typical of a monotonic deformation. A transient in the work hardening occurs after the change of strain path. Results from previous research on mechanical behaviour after reloading have been summarised as follows (Ghosh and Backofen, 1973; Wagoner and Laukonis, 1983; Chung and Wagoner, 1986; Zandrahimi et al., 1989):

- low initial flow stress (compared with stress at equal equivalent strain in the monotonic path) is followed by a relatively high work hardening rate;

- high initial flow stress is followed by a low work hardening rate.

The former case corresponds mainly to strain path changes, associated with Bauschinger experiments (inversion of loading conditions). However, most behaviour in complex strain paths involves the second case, where the active slip systems change partially or totally after reloading. When a strain path change occurs, the 
reloading behaviour of metals depends on the amplitude in the change of strain path, but is independent of the nature of prestrain conditions, such as: uniaxial tension, rolling, equibiaxial stretching and shear (Vieira et al., 1990). An equation has recently been proposed, to describe the mechanical behaviour of prestrain metals samples (Fernandes et al., 1998). This equation was deduced from the one commonly used in monotonic deformation: the Swift law (Swift, 1952). The adjustable parameters determined by fitting this equation to the experimental curve without prestrain, remain the same in the modified law. This one contains a new parameter, which depends on the path change.

Several theoretical and numerical approaches ((Hart, 1970; Miles, 1975; Jonas and Baudelet, 1977; Ghosh, 1977; Hutchinson and Neale, 1977; Ghosh, 1980; Lin et al., 1981; Barata da Rocha et al., 1985; Lian and Baudelet, 1986; Tvergaard, 1993; Rees, 1994; Esche and Shivpuri, 1998) for example), including those involving instability and flow localisation, have been used to study ductility. Most of these instability and flow localisation analysis are based on the assumption that the samples have an initial small imperfection, which is source of non-uniformity of deformation along the gauge length of the sample. As has been highlighted, imperfections grow at an increasing rate from the start of plastic deformation. The present work is an analytical study of the tensile ductility in reloading; in particular, the experimentally observed non-uniform deformation is studied (Fernandes and Vieira, 1997). This was achieved by using a simplified macroscopic two-zone model, to simulate initial material imperfection. The validation of the results was made by performing experimental tensile tests on non-prestrained copper and steel samples, with imperfections of different sizes. The mechanical behaviour of copper and steel sheets during tension of cold-worked samples is examined and the influence of the presence of geometrical defects in the prestrained samples is discussed. The importance of mechanical behaviour without prestrain is considered. In addition, the influence of the type of change of strain path and the level of the prestrain is also studied. The development of diffuse necking is also analysed as a consequence of instability corresponding to the maximum load.

\section{Analysis}

\subsection{Strain hardening law}

In this paper, material flow behaviour in simple strain paths is described by the Swift equation. In the case of reloading, the modified form of the Swift law for prestrained materials, as previously proposed, is used. In its original formulation, the Swift equation can be written as (Swift, 1952):

$$
\sigma=k\left(\varepsilon_{0}+\varepsilon\right)^{n}
$$

where $\sigma$ and $\varepsilon$ are the equivalent stress and plastic strain; $k, n$ and $\varepsilon_{0}$ are constants for a particular material, determined in uniaxial tension tests. Based on this, a modified equation for prestrained materials has been formulated as follows (Fernandes et al., 1998):

$$
\sigma=k\left[g\left(\varepsilon_{0}+\bar{\varepsilon}_{\mathrm{p}}\right)+h \varepsilon\right]^{n}
$$

In this equation $\bar{\varepsilon}_{\mathrm{p}}$ is the von Mises equivalent prestrain value and $\varepsilon$ is now the plastic strain in reloading. The parameter $g$ represents the alteration of the reloading yield stress after the path change ( $g=1$ in its absence), and $h$ characterises the work-hardening behaviour after reloading, when compared with the one in the simple strain path ( $h=1$ in this case). 


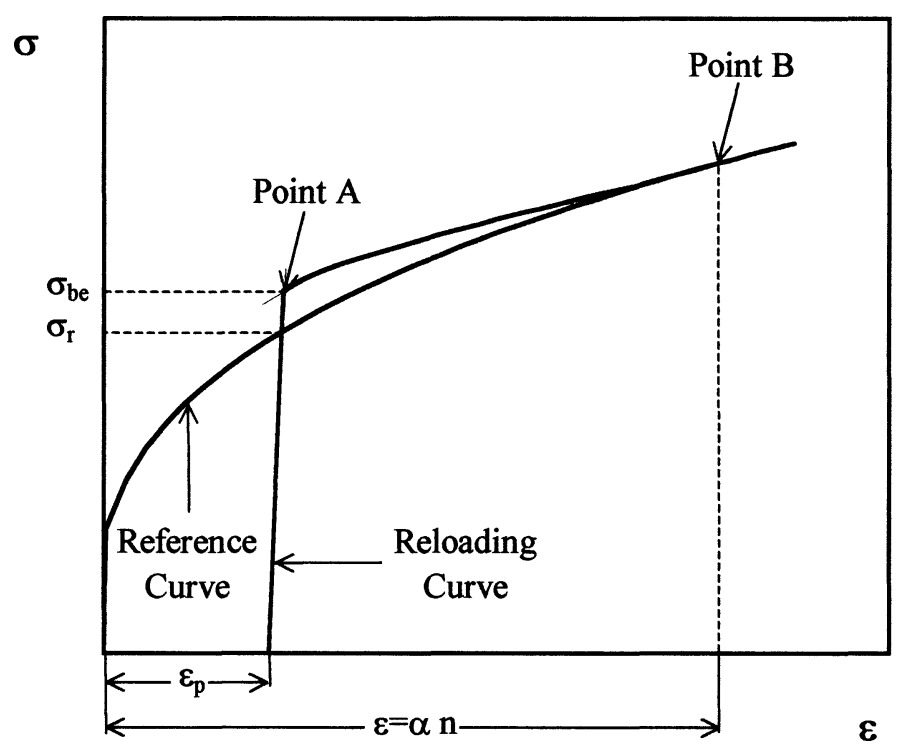

Figure 1. Schematic representation of true stress $(\sigma)$ - true strain $(\varepsilon)$ curves in tension without prestrain and after a prestrain value to $\bar{\varepsilon}_{\mathrm{p}}$.

The above equation can have a formulation identical to that of the Swift law as was originally formulated, for simple strain paths:

$$
\sigma=k h^{n}\left[\frac{g}{h}\left(\varepsilon_{0}+\bar{\varepsilon}_{\mathrm{p}}\right)+\varepsilon\right]^{n}=k^{*}\left(\varepsilon_{0}^{*}+\varepsilon\right)^{n}
$$

where $k^{*}=k h^{n}$ and $\varepsilon_{0}^{*}=(g / h)\left(\varepsilon_{0}+\bar{\varepsilon}_{\mathrm{p}}\right)$. In this context, the term $\bar{\varepsilon}_{\mathrm{p}}^{*}=(g / h) \bar{\varepsilon}_{\mathrm{p}}$, in some way, represents the plastic prestrain value adjusted to the path change.

Experimental evidence allows us to describe the strain hardening behaviour in reloading as shown in figure 1 (the reference curve, without prestrain, is also shown for comparison). In this figure $\sigma_{\text {be }}$ (the stress at point $A$ ) is the back-extrapolated stress, measured on the reloading curve $\left(\sigma_{\mathrm{be}}=\sigma(\varepsilon=0)\right)$ and $\sigma_{\mathrm{r}}$ is the reference stress, measured at the same equivalent strain on the curve without prestrain $\left(\sigma_{\mathrm{r}}=\sigma\left(\varepsilon=\bar{\varepsilon}_{\mathrm{p}}\right)\right)$. The term $\left(\sigma_{\mathrm{be}} / \sigma_{\mathrm{r}}\right)$ is called the normalised reloading yield stress (Raphanel et al., 1986). The parameter $\alpha$, as shown in figure 1 characterises the influence of the strain path change on the work-hardening behaviour in reloading ( $\alpha$ is such that the two curves meet at point $B$ ).

It is now possible to determine $g$ and $h$ as follows:

$$
g=\left(\sigma_{\mathrm{be}} / \sigma_{\mathrm{r}}\right)^{1 / n}
$$

and

$$
h=\frac{\left(\varepsilon_{0}+\alpha n\right)-\left(\sigma_{\mathrm{be}} / \sigma_{\mathrm{r}}\right)^{1 / n}\left(\varepsilon_{0}+\bar{\varepsilon}_{\mathrm{p}}\right)}{\alpha n-\bar{\varepsilon}_{\mathrm{p}}} .
$$

Equations (3), (4) and (5) model the material behaviour in reloading for several complex strain paths. Thus, it is necessary to know the monotonic behaviour, which allows us to determine the values of the parameters $k$, $n$ and $\varepsilon_{0}$, and to define the normalised reloading yield stress $\left(\sigma_{\mathrm{be}} / \sigma_{\mathrm{r}}\right)$. The parameter $\alpha$ was found to be equal to 0.8 , in agreement with experimental results for copper and steel (Fernandes et al., 1998). 
Similarly to other cases ((Ghosh, 1977; Barata da Rocha et al., 1985) for example), a constitutive law, which considers the strain rate sensitivity can be formulated, as follows:

$$
\sigma=k^{*}\left(\varepsilon_{0}^{*}+\varepsilon\right)^{n} \dot{\varepsilon}^{m}
$$

This equation is obtained from Eq. (3), assuming that the strain rate sensitivity coefficient $m$ is not dependent on the path change, i.e. $m$ is unique for a given material.

\subsection{Strain distribution relations}

During the tensile deformation of metals, non-uniform strain distribution can be observed. In the case of complex strain paths, experimental results clearly show that the deformation in tension after path change often starts from a limited region in the sample, spreading over the length of the sample before necking takes place. The strain gradient increases during deformation and is related to the presence of fluctuations in the cross section area of the sample, occuring even when allowed by standardisation. The non-uniform deformation depends on the prestrain value and path change, which determines subsequent work-hardening behaviour, for a given material. The effect of the area fluctuations becomes important for materials with relatively low work-hardening rate and high reloading stress (Fernandes and Vieira, 1997). The way in which the strain gradient develops as a function of the size of the geometrical defect and of the work hardening behaviour after path change, will be subsequently discussed. The analysis carried out here follows the one used by Ghosh (1977) to study the tensile instability and necking development in materials with strain hardening and strain rate sensitivity. It is considered that the specimen has a pre-existing geometrical imperfection. This means a simplified model of a two zones sample. It considers that the strain path is in tension for both regions. Tensile deformation is defined as stable as long as it is accompanied by load rise, even though the geometrical imperfection may be growing during this stage. The onset of instability is therefore at the maximum load. It should be noted that the present model does not take into account the localised necking process, in pure uniaxial tension, which occurs along a line inclined to that normal to the tensile direction. However, it can predict, with no major error, the macroscopic strain levels in both regions during the development of diffuse necking, along the tensile axis, i.e. clearly after the Considère point. Moreover, this is a reasonable approach, regarding the growth of the imperfection in the case of prestrain materials and its dependency on the combined effects of the strain hardening parameter, prestrain level and type of path change.

The initial cross-section of the imperfection is assumed to be less than the one outside by a fraction $f_{0}=S_{0}^{\text {in }} / S_{0}^{\text {out }}$, where $S_{0}^{\text {in }}$ and $S_{0}^{\text {out }}$ are the initial cross-section areas of the tensile sample, inside and outside the imperfection region, respectively. Under these conditions, the applied force $F$ at the tensile sample is given by the equation:

$$
F=\sigma^{\text {in }} S^{\text {in }}=\sigma^{\text {out }} S^{\text {out }}
$$

where $\sigma^{\text {in }}$ and $\sigma^{\text {out }}$ are the true stress inside and outside the imperfection, and $S^{\text {in }}$ and $S^{\text {out }}$ are the current cross-section areas in the same regions, respectively. The following conditions can now be written:

$$
\begin{aligned}
& S^{\text {in }}=S_{0}^{\text {in }} \exp \left(-\varepsilon_{\text {in }}\right), \\
& S^{\text {out }}=S_{0}^{\text {out }} \exp \left(-\varepsilon_{\text {out }}\right),
\end{aligned}
$$

where $\varepsilon_{\text {in }}$ and $\varepsilon_{\text {out }}$ are the strain values inside and outside the imperfection region. Equation (7) can now be written as follows:

$$
f_{0} \sigma^{\text {in }} \exp \left(-\varepsilon_{\text {in }}\right)=\sigma^{\text {out }} \exp \left(-\varepsilon_{\text {out }}\right)
$$


Considering Eq. (6) to characterize the material behaviour in reloading, Eq. (9) becomes:

$$
f_{0}\left(\varepsilon_{0}^{*}+\varepsilon_{\text {in }}\right)^{n}\left(\dot{\varepsilon}_{\text {in }}\right)^{m} \exp \left(-\varepsilon_{\text {in }}\right)=\left(\varepsilon_{0}^{*}+\varepsilon_{\text {out }}\right)^{n}\left(\dot{\varepsilon}_{\text {out }}\right)^{m} \exp \left(-\varepsilon_{\text {out }}\right)
$$

or, in the differential form:

$$
\left[f_{0}\left(\varepsilon_{0}^{*}+\varepsilon_{\text {in }}\right)^{n} \exp \left(-\varepsilon_{\text {in }}\right)\right]^{1 / m} \mathrm{~d} \varepsilon_{\text {in }}=\left[\left(\varepsilon_{0}^{*}+\varepsilon_{\text {out }}\right)^{n} \exp \left(-\varepsilon_{\text {out }}\right)\right]^{1 / m} \mathrm{~d} \varepsilon_{\text {out }} .
$$

Equation (11) can be solved with resource to numerical methods such as the Newton-Raphson iterative technique, used in this work. The strain increments used are $\Delta \varepsilon_{\text {in }}=0.001$ and it was considered that:

$$
\varepsilon_{\text {in }}=\int_{\varepsilon_{\text {in }}^{c}}^{\varepsilon_{\text {in }}} \mathrm{d} \varepsilon_{\text {in }} \quad \text { and } \quad \varepsilon_{\text {out }}=\int_{0}^{\varepsilon_{\text {out }}} \mathrm{d} \varepsilon_{\text {out }},
$$

$\varepsilon_{\text {in }}^{c}$ being an estimated value of $\varepsilon_{\text {in }}$, for which deformation starts in the outside region. The value of $\varepsilon_{\text {in }}^{c}$ can be obtained using Eq. (9) for $\varepsilon_{\text {out }}$ equal to zero, which leads to the condition for $\varepsilon_{\text {in }}^{c}$ :

$$
\varepsilon_{\text {in }}^{c}=\varepsilon_{0}^{*}\left\{\left[\frac{\exp \left(\varepsilon_{\text {in }}^{c}\right)}{f_{0}}\right]^{1 / n}-1\right\} .
$$

In a recent work (Fernandes and Vieira, 1997), non-uniform deformation was clearly observed, by optical microscopy, during the reloading of a sample, which was previously prestrained in rolling and polished. It was concluded that, the fluctuations in the cross-sectional area become important for materials with a relatively low work-hardening rate and high reloading stress. In fact, the cross-section area inside the imperfection deforms faster than outside. Since an area difference spreads over different axial distances, the gradient of imperfection profile may be a good indicator of necking growth (Ghosh, 1977). An analytical way to study this effect is to follow the evolution, during deformation, of the difference between the square root of the cross-sectional areas in both regions $\left(\sqrt{S^{\text {out }}}-\sqrt{S^{\text {in }}}\right)$ with respect to their current separation distance $\Delta x$. This can be approximately determined by (Ghosh, 1977):

$$
\Delta x=\Delta x_{0} \frac{\exp \left(\varepsilon_{\text {out }}\right)+\exp \left(\varepsilon_{\text {in }}\right)}{2} .
$$

Thus, the neck profile gradient can be defined, for example:

$$
\frac{\sqrt{\Delta S}}{\Delta x}=\frac{\sqrt{S_{0}^{\text {out }}}}{\Delta x_{0}}\left\{\frac{2\left[\exp \left(\frac{-\varepsilon_{\text {out }}}{2}\right)-\sqrt{f_{0}} \exp \left(\frac{-\varepsilon_{\text {in }}}{2}\right)\right]}{\exp \left(\varepsilon_{\text {out }}\right)+\exp \left(\varepsilon_{\text {in }}\right)}\right\} .
$$

Next, this method is tested, to study the effect of the strain path change (by means of the value of the respective reloading yield stress $\left.\left(\sigma_{\mathrm{be}} / \sigma_{\mathrm{r}}\right)\right)$ on tensile instability, and diffuse necking development. In order to do this, a two region imperfect sample is used. After this, the results obtained after reloading are discussed, taking the respective flow behaviour into account, as described below.

\section{Experimental validation}

Oxygen-free copper $(99.95 \% \mathrm{Cu})$ and steel sheets, $1 \mathrm{~mm}$ thick, as-received under cold-rolled and annealed conditions, were used in this research. The two sheets present equiaxed grain with approximately the same 


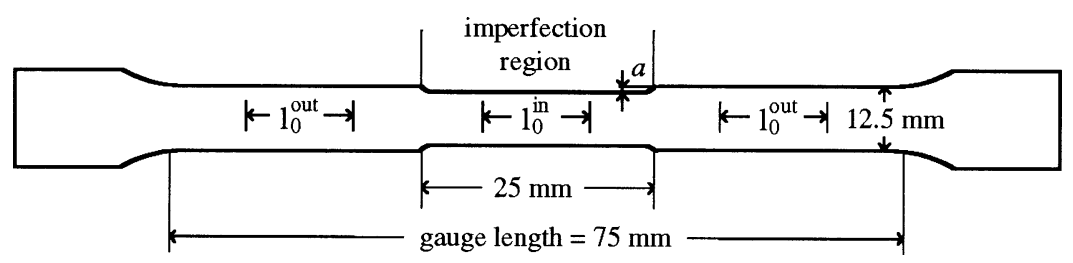

Figure 2. Schematic representation of the specimen with cross section imperfection used for experimental tests and the simulation of samples without prestrain. The value of $a$ was considered as equal to $0.25,0.50$ and $1.00 \mathrm{~mm}$, which corresponds to, $f_{0}=0.96,0.92$ and 0.84 , respectively.

mean grain size: 20 and $15 \mu \mathrm{m}$, respectively. Samples of both sheets, copper and steel, were cut accordingly with an ISO 50 (gauge length and width of $75 \mathrm{~mm}$ and $12.5 \mathrm{~mm}$, respectively), their axis being normal to the rolling direction. Tensile tests were performed at room temperature, using an initial strain rate $\dot{\varepsilon}=7 \times 10^{4} \mathrm{~s}^{-1}$. The true stress $(\sigma)$ - true strain $(\varepsilon)$ curves were obtained in tension, for copper and steel sheets. The fitting of these curves by Eq. (1) allowed us to determine the adjustable parameters, as follows:

- copper sheet: $k=510 \mathrm{MPa}, n=0.41$ and $\varepsilon_{0}=0.0150$,

- steel sheet: $k=585 \mathrm{MPa}, n=0.25$ and $\varepsilon_{0}=0.0075$.

For both sheets, copper and steel, some other samples were cut also accordingly using an ISO 50, but with geometrical imperfection. This consists of a reduction of width in the central area, which is a length of $1 / 3$ of the total gauge length of the sample, as shown in figure 2. The consequent reduction of the section is characterised by the dimension of $a$ as follows: $a=0.25,0.5$ and $1.0 \mathrm{~mm}$, which corresponds to fraction areas $f_{0}=0.96$, 0.92 and 0.84 , respectively. The tensile tests were performed using an Instron machine, equipped with a 50 $\mathrm{mm}$ strain gauge and using an initial strain-rate as described above, for the uniform samples. In the case of the samples with $a=0$, the strain amounts were deduced from extensometer data. A microcomputer was interfaced with the tensile testing machine and signals from load, and extension were stored and analysed digitally. For the cases of $a \neq 0$ the plastic strain measurement was performed in both regions of the samples. Previous to deformation, reference lengths $l_{0}^{\text {in }}$ and $l_{0}^{\text {out }}$, inside and outside the imperfection, respectively, were marked in the samples (figure 2). It was then possible to follow the evolution of the deformation in both regions during the test. In order to do this, the reference lengths were measured before and after several steps of deformation, using a travelling microscope, which had an accuracy of $1 \mu \mathrm{m}$.

The results are shown in figure 3, considering $\varepsilon_{\text {in }}$ as a function of $\varepsilon_{\text {out }}$. Whatever the value of the imperfection parameter $a=0.25,0.50$ or $1.00 \mathrm{~mm}$ ( $f_{0}=0.96,0.92$ and 0.84 , respectively), the strain gradient between the two regions increases during deformation. In figure 3, the results of numerical computation are also shown. Based on Eq. (11), they allow comparison with the experimental results. The numerical computation results are considered in two cases: taking $m=0$ and in the case of $m=0.012$, as indicated for steel and copper. The case $m=0.012$ shows perfect agreement with the experimental results. The strain-rate hardening exponent $m$, has an effect of slowing the growth rate of the imperfection, as was argued by Ghosh (1977). For this reason, we consider the following value of $m$ as always being equal to 0.012 , for both materials, which corresponds to the experimental values usually indicated (Barata da Rocha et al., 1985; Gracio et al., 1987).

In figure 4 , the numerical computation results for the cases of $f_{0}=0.995,0.990,0.985$, and 0.980 are shown. These cases are allowed by standardisation (the last one being near the allowable limit). The case of $f_{0}=0.96$ is also considered, in order to show a case for which experimental results have been obtained (for $f_{0}$ lower than 0.96 , the experimental measurement of the dimensions of the sample becomes relatively inexact; this is the reason why these cases were not experimentally considered). In all cases, the profile gradient $(\sqrt{\Delta S} / \Delta x)$ develops slowly, at the beginning, and increases with deformation. The imperfection 

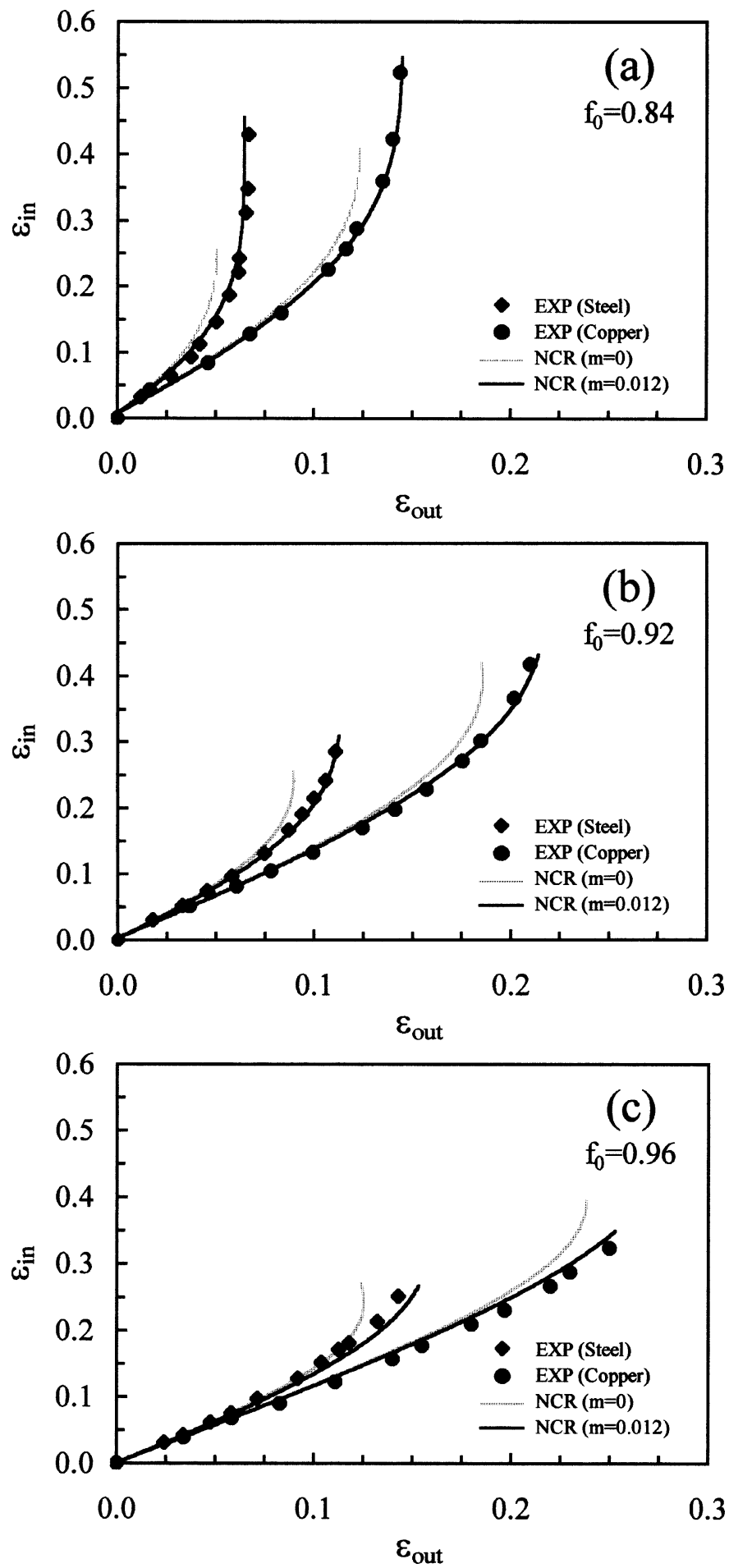

Figure 3. Comparison between experimental (EXP) and numerical computational results (NCR), for copper and steel, on the evolution of the strain inside the imperfection region as a function of the strain outside this region, for: (a) $f_{0}=0.84$; (b) $f_{0}=0.92$ and (c) $f_{0}=0.96$. 

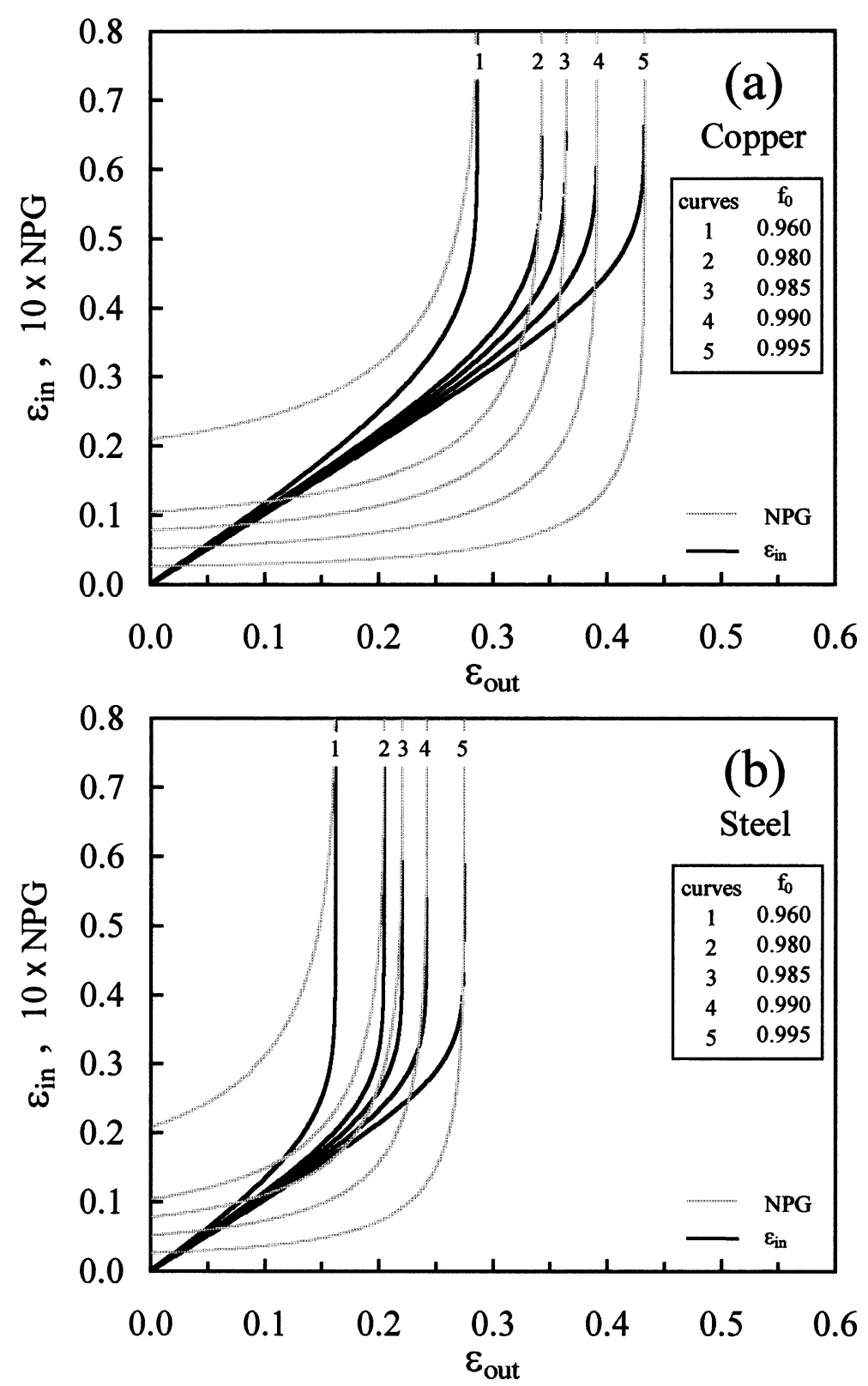

Figure 4. Numerical computational results showing the strain within the imperfection and the necking profile gradient, as functions of strain outside imperfection. The necking profile gradient is normalised with respect to the initial gradient: NPG $=\left(\sqrt{S^{\text {out }}} / \Delta x\right) /\left(\sqrt{S_{0}^{\text {out }}} / \Delta x_{0}\right)$. The size of the imperfection is $f_{0}=0.995, f_{0}=0.990, f_{0}=0.985, f_{0}=0.980$ and $f_{0}=0.960$. Both materials are considered: (a) copper and (b) steel.

growth kinetics are influenced by the values of $n$ and $f_{0}$. The increasing value of the strain-hardening exponent, $n$, and the increasing value of $f_{0}$ also slow the spread of the imperfection.

The above results show that, the strain value attained in the outside region is dependent on the imperfection size. We believe that in most of the cases, the tensile samples present imperfections with $f_{0}=0.99$. In fact, this value represents approximately half of the imperfection allowed by standardisation. Moreover, in general, the uniform strain obtained by tension is close to the value of the hardening coefficient $n$, at least for low 
strain rates (usually used for classical tensile tests). Or, in the case of $f_{0}=0.99$, the strain in the region outside the imperfection has approximately the same maximum strain value as the hardening coefficient $n$, for both materials (maximum $\varepsilon_{0}$ equal to 0.39 for copper $(n=0.41)$ and 0.24 to steel $(n=0.25)$ ). So, $f_{0}=0.99$ must be representative of most cases of tensile tests and it will be always considered in the following discussion.

\section{Flow localisation in prestrained materials}

In tension, the phenomenon of flow localisation is recognised as a precursor to failure. During complex strain paths, an early flow localisation is often observed ((Vieira et al., 1990; Schmitt et al., 1991; Fernandes et al., 1993) for example). In this context, it is useful to emphasise the correlation between the hardening behaviour and the strain distribution along the subsequent path. The effect of the strain path change can be analysed by means of the parameter $\left(\sigma_{\mathrm{be}} / \sigma_{\mathrm{r}}\right)$. This parameter characterises the strain path change: $\left(\sigma_{\mathrm{be}} / \sigma_{\mathrm{r}}\right)=1$ when there is no strain path change and its value can attain 1.12 or even 1.15 for the harder strain path change as, for example, in tension normal to previous rolling (Fernandes and Vieira, 1997). Latent hardening effects can explain the behaviour in reloading, particularly, the range of values of $\left(\sigma_{\mathrm{be}} / \sigma_{\mathrm{r}}\right)$. In fact, the differences between monotonic and subsequent work-hardening behaviour are related to different microstructural evolutions at the beginning of reloading. These are dependent on the prestrain mode and path change. Microstructural analyses during reloading confirmed this point (Schmitt et al., 1991).

The present study concerns copper and steel sheets, whose flow behaviour laws are described in the former paragraph ( $n=0.41$ for copper and $n=0.25$ for steel; $m$ is considered equal to 0.012 for both materials). The Swift equation (Eq. (1)) describes the behaviour in simples strain path, and the Eq. (6) describes the behaviour in reloading. The numerical computation method proposed by Ghosh (1977) was adapted for the present case (Eq. (10) to (15)), as has been described above.

In figures 5 and 6 numerical computational results show the evolution of the strain within the imperfection, and the necking profile gradient. These are demonstrated as functions of strain outside imperfection, during deformation in reloading, for different $\left(\sigma_{\mathrm{be}} / \sigma_{\mathrm{r}}\right)$ values. For both materials, copper (figure 5) and steel (figure 6 ), two prestrain values are presented. For the low prestrain values, the curves are not significantly influenced by the $\left(\sigma_{\mathrm{be}} / \sigma_{\mathrm{r}}\right)$ value. When the prestrain is higher (at about half the value of $n$ ), the prestrain value strongly influences the evolution of the strain within the imperfection and the necking profile gradient.

A final exercise, shown in figures 7 and 8, explains the conjoint effect of prestrain and strain path change on strain localisation in reloading. This is evaluated by the residual strain defined by (i) the maximum strain attainned in the outside region $\varepsilon_{\text {out }}^{\text {res }}$, and (ii) the strain inside the imperfection region up to unstable flow $\varepsilon_{\text {in }}^{\text {res }}$, such that the critical value $\mathrm{d} \varepsilon_{\text {out }} / \mathrm{d} \varepsilon_{\text {in }}$ is chosen equal to 0.1 . Both figures show the evolution of the residual strain as a function of $\left(\sigma_{\mathrm{be}} / \sigma_{\mathrm{r}}\right)$, for six cases of prestrain values. For low prestrain values ( $\bar{\varepsilon}_{\mathrm{p}}$ up to 0.06 , for copper, and 0.03 , for steel) a homogeneous deformation is observed in the tensile sample, when a large amount of strain is being applied. This effect is only slightly dependent on the strain path change through the value of $\left(\sigma_{\mathrm{be}} / \sigma_{\mathrm{r}}\right)$. For higher prestrain values rapid strain localisation within the imperfection is often observed. Finally, the comparison between both materials allows us to conclude that the imperfection growth kinetics in prestrained samples is highly influenced by the work-hardening parameter $n$.

\section{Conclusion}

A simplified analysis was used to model the behaviour in tension of a metallic specimen, with a geometrical imperfection. The flow behaviour is described by a Swift law equation including strain-rate sensitivity. A 

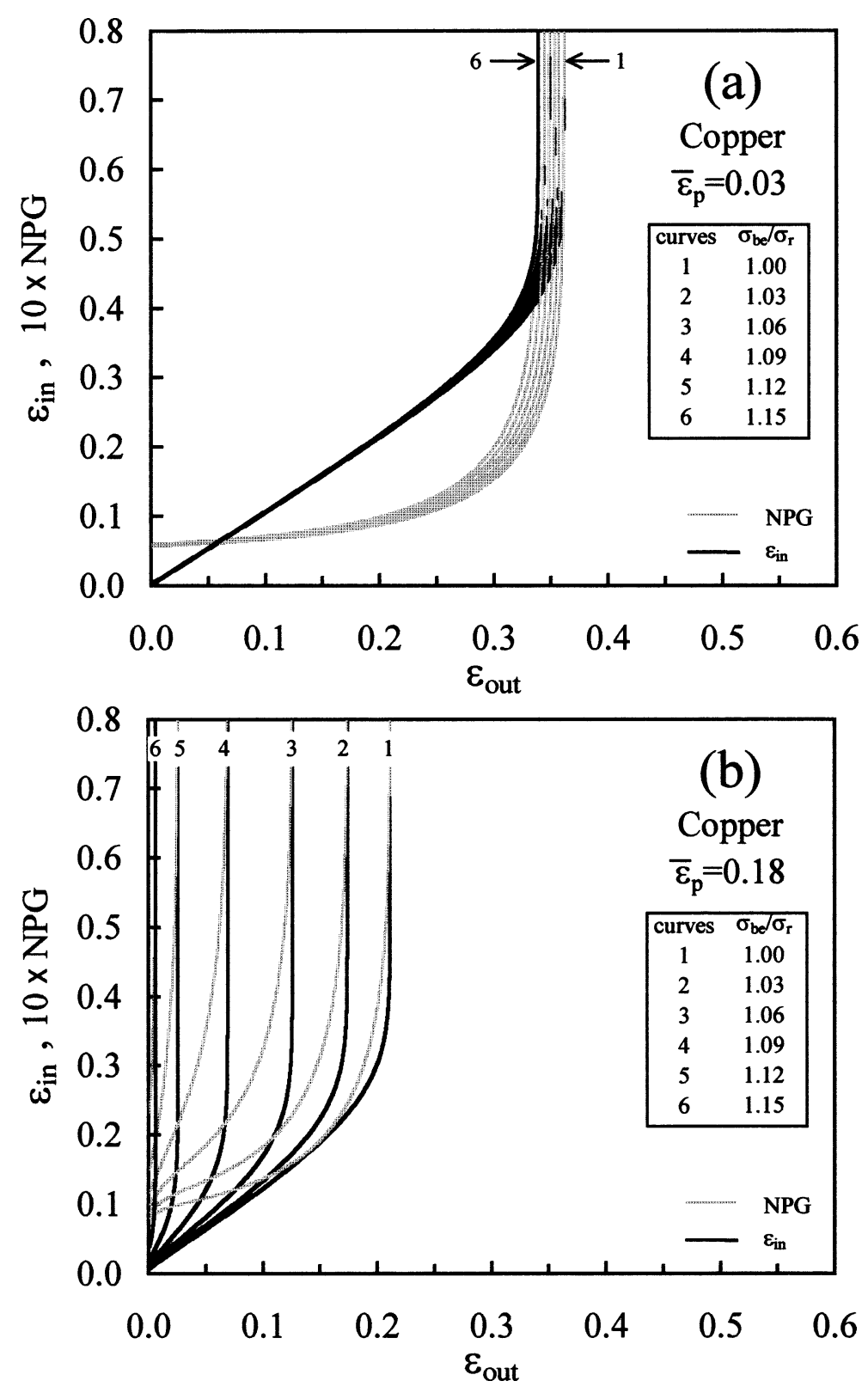

Figure 5. Numerical computational results showing the strain within the imperfection and the necking profile gradient, as functions of strain outside imperfection, during the deformation of copper. The necking profile gradient is normalised with respect to the initial gradient: NPG $=$ $\left(\sqrt{S^{\text {out }}} / \Delta x\right) /\left(\sqrt{S_{0}^{\text {out }}} / \Delta x_{0}\right)$. The size of the imperfection is $f_{0}=0.99$ and the change of strain path is such that: (a) $\bar{\varepsilon}_{\mathrm{p}}=0.03$ and (b) $\bar{\varepsilon}_{\mathrm{p}}=0.18$.

modified law was used for prestrained materials, which incorporate the plastic prestrain value adjusted to the path change. Experimental results obtained in simple tension of samples with large imperfection and in prestrained samples with imperfections as allowed by standardisation, were used to validate the method used. The main study concerns the combined effects of the strain-hardening exponent, the prestrain value and the type of path change. In this context, the following conclusion can be drawn: 

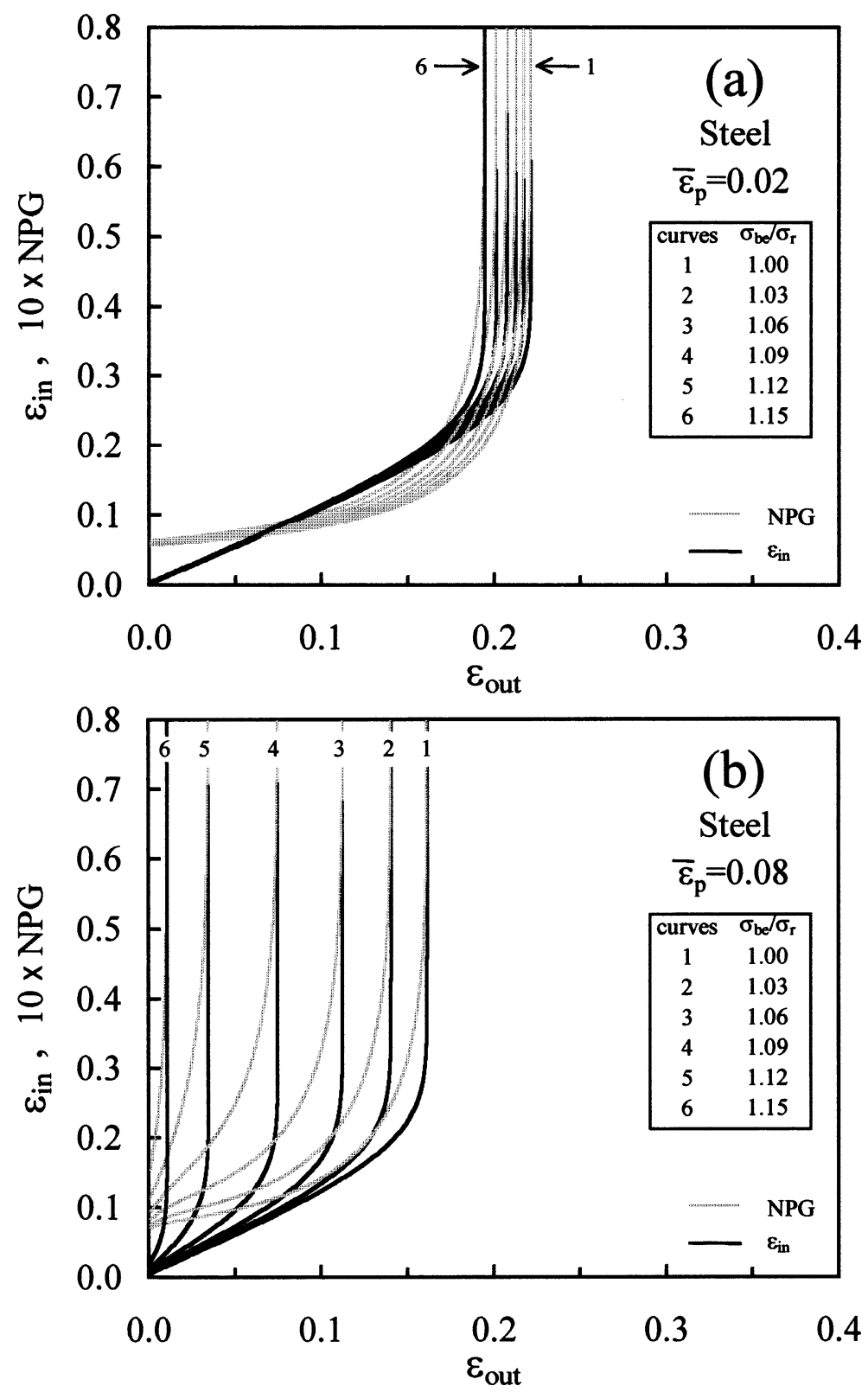

Figure 6. Numerical computational results showing the strain within the imperfection and the necking profile gradient, as functions of strain outside imperfection, during the deformation of steel. The necking profile gradient is normalised with respect to the initial gradient: NPG $=$ $\left(\sqrt{S^{\text {out }}} / \Delta x\right) /\left(\sqrt{S_{0}^{\text {out }}} / \Delta x_{0}\right)$. The size of the imperfection is $f_{0}=0.99$ and the change of strain path is such that: (a) $\bar{\varepsilon}_{\mathrm{p}}=0.02$ and $(\mathrm{b}) \bar{\varepsilon}_{\mathrm{p}}=0.08$.

- The evolution of the strain in both zones of the sample and the strain saturation in the region outside the imperfection due to the onset of necking, can be well predicted by the model, as was experimentally confirmed.

- The model predicts that the imperfection growth kinetics is influenced by the strain hardening exponent $n$, obtained in simple strain path. The spread of the imperfection slows down with increasing $n$ values, even after path change. 


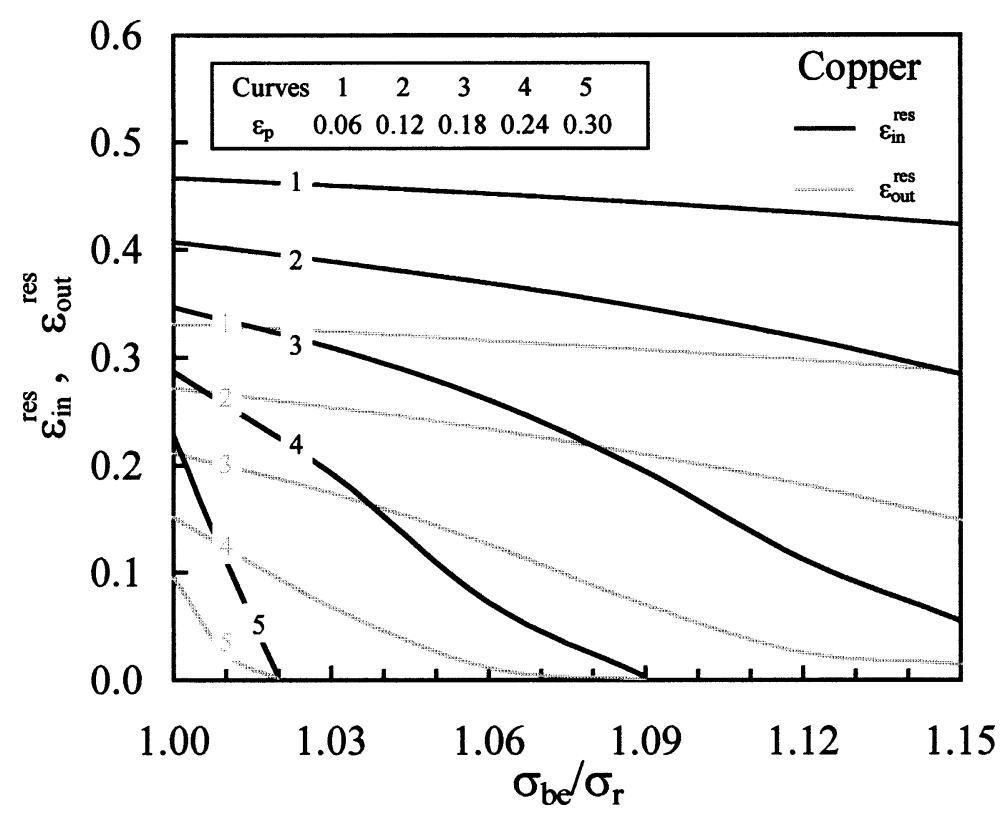

Figure 7. Evolution of the residual strain in the outside region $\left(\varepsilon_{\mathrm{out}}^{\mathrm{res}}\right)$, in the reloading of copper, with the parameter $\left(\sigma_{\mathrm{be}} / \sigma_{\mathrm{r}}\right)$, for different cases of prestrain values: $\bar{\varepsilon}_{\mathrm{p}}=0.06,0.12,0.18,0.24$ and 0.30 . For the same cases, the evolution of the residual strain inside the imperfection region up to unstable flow $\left(\varepsilon_{\text {in }}^{\text {res }}\right)$ is also shown. This is defined by the critical value $\mathrm{d} \varepsilon_{\text {out }} / \mathrm{d} \varepsilon_{\text {in }}$ chosen equal to 0.1 .

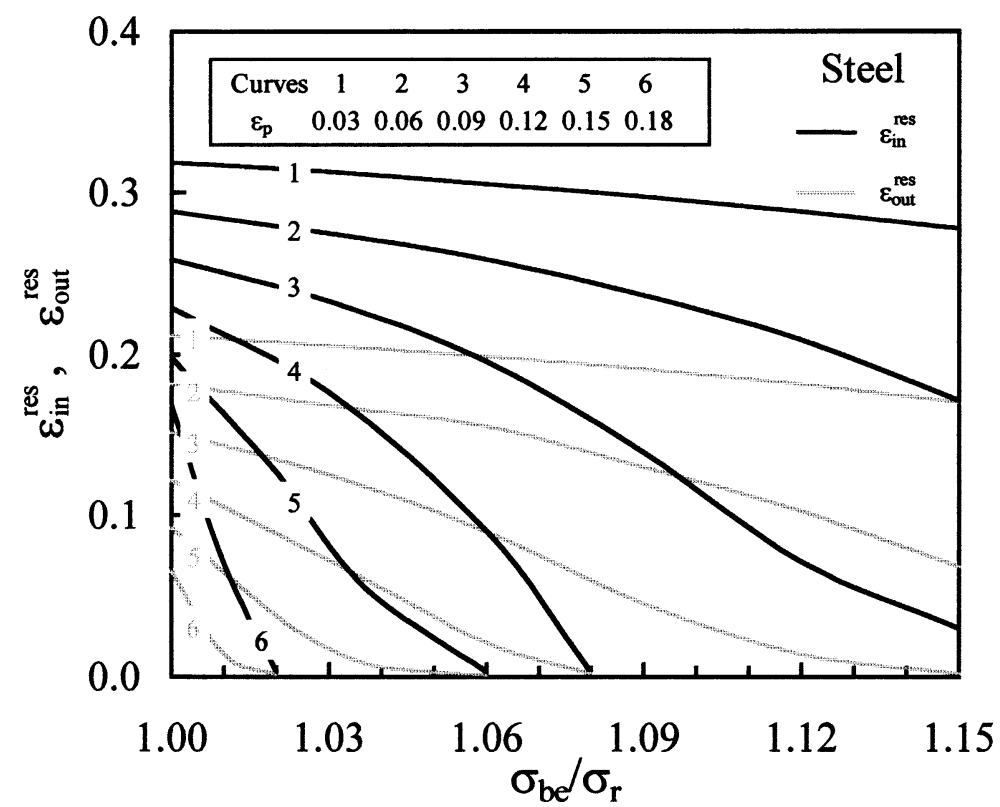

Figure 8. Evolution of the residual strain in the outside region $\left(\varepsilon_{\text {out }}^{\text {res }}\right)$, in reloading of steel, with the parameter $\left(\sigma_{\text {be }} / \sigma_{\mathrm{r}}\right)$, for different cases of the prestrain values: $\bar{\varepsilon}_{\mathrm{p}}=0.03,0.06,0.09,0.12,0.15$ and 0.18 . For the same cases, the evolution of the residual strain inside the imperfection region up to unstable flow $\left(\varepsilon_{\text {in }}^{\text {res }}\right)$ is also shown. This is defined by the critical value $\mathrm{d} \varepsilon_{\text {out }} / \mathrm{d} \varepsilon_{\text {in }}$ chosen equal to 0.1 . 
- For low prestrain values (less than $n / 3$ ) the strain path change has a minor effect on strain localisation. In this case, a homogeneous deformation is always observed in the tensile sample, when a large amount of strain is being applied. For higher prestrain values, the strain distribution behaviour is quite dependent on the value $\left(\sigma_{\mathrm{be}} / \sigma_{\mathrm{r}}\right)$ and therefore, on the strain path change.

\section{Acknowledgement}

The authors are indebted to the Portuguese Science and Technology Foundation and Program PRAXIS XXI, for financial support.

\section{References}

Barata da Rocha A., Barlat F., Jalinier J.M., 1985. Prediction of the forming limit diagrams of anisotropic sheets in linear and non-linear loading. Mater. Sci. Eng. 68, 151-164.

Chung K., Wagoner R.H., 1986. Effect of stress-strain-law transients on formability, Metall. Trans. A 17, 1001-1009.

Esche S.K., Shivpuri R., 1998. A consistent criterion for diffuse necking in sheet metals using Hill's 1979 yield surface. J. Eng. Mater. Technol. 120, $177-182$.

Fernandes J.V., Gracio J.J., Schmitt J.-H, 1993. Grain size effect on the microstructural evolution of copper deformed in rolling-tension. In: C. Teodosiu, J.L. Raphanel and F. Sidoroff (Eds), Large Plastic Deformations: Fundamental Aspects and Applications to Metal Forming, A.A. Balkema, Rotterdam, pp. 219-228.

Fernandes J.V., Vieira M.F., 1997. Strain distribution in copper tensile specimens prestrained in rolling. Metall. Mater. Trans. A 28, $1169-1179$.

Fernandes J.V., Rodrigues D.M., Menezes L.F., Vieira M.F., 1998. A modified Swift law for prestrained materials. Int. J. Plasticity 14, 537-550.

Ghosh A.K., Backofen W.A., 1973. Strain hardening and instability in biaxially streched sheets. Metall. Trans. 4, 1113-1123.

Ghosh A.K., 1977. Tensile instability and necking in materials with strain hardening and strain-rate hardening. Acta Metall. 25, $1413-1424$.

Ghosh A.K., 1980. A physically-based constitutive model for metal deformation. Acta Metall. 28, 1443-1465.

Gracio J.J., Fernandes J.V., Barata da Rocha A., 1987. Theoretical prediction of the limit curves for simulation of plastic instability. In: M. Predeleanu (Ed.), Computational Methods for Predicting Material Processing Defects, Elsevier, Amsterdam, pp. 161-170.

Hart E.W., 1970. A phenomelological theory for plastic deformation of polycrystalline metals. Acta Metall. 18, 599-610.

Hutchinson J.W., Neale K.W., 1977. Influence of strain-rate sensitivity on necking under uniaxial tension. Acta Metall. 25, 839-846.

Jonas J.J., Baudelet B., 1977. Effect of crack and cavity generation on tensile stability. Acta Metall. 25, 43-50.

Lian J., Baudelet B., 1986. Necking development and strain to fracture under uniaxial tension. Mater. Sci. Eng. 84, 157-162.

Lin I.-H., Hirth J.P., Hart E.W., 1981. Plastic instability in uniaxial tension tests. Acta Metall. 29, 819-827.

Menezes L.F., Fernandes J.V., Rodrigues D.M., 1999. Numerical simulation of tensile tests of prestrained sheets. Mater. Sci. Eng. A 264, 130-138.

Miles J.P., 1975. The initiation of necking in rectangular elastic/plastic specimens under uniaxial and biaxial tension. J. Mech. Phys. Solids 23, 197-213.

Raphanel J.L., Schmitt J.-H., Baudelet B., 1986. Effect of a prestrain on the subsequent yielding of a low carbon steel sheets: experiments and simulations. Int. J. Plast. 2, 371-378.

Rees D.W.A., 1994. Equivalence and instability correlations for isotropic and anisotropic sheet plasticity. J. Mater. Process. Technol. 40, $173-194$.

Schmitt J.-H., Fernandes J.V., Gracio J.J., Vieira M.F., 1991. Plastic behaviour of copper sheets during sequential tension tests. Mater. Sci. Eng. A $147,143-154$.

Swift H.W., 1952. Plastic instability under plane stress. J. Mech. Phys. Solids 1, 1-18.

Tvergaard V., 1993. Necking in tensile bars with rectangular cross-section. Comp. Meth. in Appl. Mech. and Engn. 103, $273-290$.

Vieira M.F., Schmitt J.-H., Gracio J.J., Fernandes J.V., 1990. The effect of strain path change on the mechanical behaviour of copper sheets. J. Mater. Process. Technol. 24, 313-322.

Wagoner R.H., Laukonis J.V., 1983. Plastic behaviour of aluminium-killed steel following plane-strain deformation. Metall. Trans. A 14, 1487-1495.

Zandrahimi M., Platias S., Price D., Barret D., Bate P.S., Roberts W.T., Wilson D.V., 1989. Effects of change in strain path on work hardening in cubic metals. Metall. Trans. A 20, 2471-2482. 